\title{
A DENOISING OF BIOMEDICAL IMAGES
}

\author{
Thanh D.N.Ha , Dvoenko S.D. ${ }^{\mathrm{a}}$ \\ a Tula State University, Institute of Applied Mathematics and Computer Sciences, Lenin ave. 92 Tula city, Russian Federation - \\ (dnhthanh@hueic.edu.vn,dsd@tsu.tula.ru)
}

\section{Commission VI, WG VI/4}

KEY WORDS: Total variation, ROF model, Gaussian noise, Poisson noise, Mixed Poisson-Gaussian noise, Image processing, Biomedical image, Euler-Lagrange equation.

\begin{abstract}
:
Today imaging science has an important development and has many applications in different fields of life. The researched object of imaging science is digital image that can be created by many digital devices. Biomedical image is one of types of digital images. One of the limits of using digital devices to create digital images is noise. Noise reduces the image quality. It appears in almost types of images, including biomedical images too. The type of noise in this case can be considered as combination of Gaussian and Poisson noises. In this paper we propose method to remove noise by using total variation. Our method is developed with the goal to combine two famous models: ROF for removing Gaussian noise and modified ROF for removing Poisson noise. As a result, our proposed method can be also applied to remove Gaussian or Poisson noise separately. The proposed method can be applied in two cases: with given parameters (generated noise for artificial images) or automatically evaluated parameters (unknown noise for real images).
\end{abstract}

\section{INTRODUCTION}

One of the important types of digital technique that has many applications in many fields of life is digital image. It is a type of a signal that is obtained from a real analogous signal by discretization and quantization. There are many devices can create digital images, such as digital camera, X-ray scanner, and so on. Ordinarily these devices can give unexpected effects. One of them is noise. Noise reduces image quality and efficiency of image processing.

The problem of noise removal from digital images is very actual today. In order to remove noise more effectively, we need to classify it. There are many types of noise, for example, Gaussian noise (almost for digital image by using digital camera), Poisson noise (for X-ray image), speckle noise (for ultrasonogram), and so on.

There are many developed strong approaches to solve noise removal problem. The approach that uses total variation (Chan, 2005; Burger, 2008; Chambolle, 2009; Xu, 2014; Rankovic, 2012; Lysaker, 2006; Li, 2006; Zhu, 2012; Tran, 2012; Getreuer, 2012; Caselles, 2011; Rudin, 1992; Chen, 2013) is well-known and very promising.

Rudin (1992) is pioneer to apply this concept. He proposed the total variation to solve many problems in image processing. Especially, he built a model to remove noise on digital images. This model is named as ROF (Rudin, 1992; Chen, 2013).

However, ROF model is usually used to remove only Gaussian noise. Of course it can also remove other types of noise, but not very effectively. Another popular noise in medical images is Poisson noise. For example, this noise appears in medical X-ray images. ROF model cannot treat this noise effectively. Therefore, Le T. (2007) developed so called modified ROF model.

Both of Gaussian and Poisson noises is popular, but their combination is also important (Luisier, 2011). This combination of noises usually appears in biomedical images, for example, in electronic microscopy images (Jezierska, 2011; Jezierska 2012).

As we talk above, ROF and modified ROF models ineffectively treat this combination. ROF model gives priority to Gaussian noise, but modified ROF model gives it to Poisson noise.

In order to treat this combination of noise, we will combine ROF model (for Gaussian noise) and modified ROF model (for Poisson noise). Our model will treat this combination by considering proportion of noise between them.

In experiments, we used a real image and add noise into them. We performed denoising of digital images by proposed method and other methods, such as ROF model, median filter (Wang, 2012) and Wiener filter (Abe, 2012). In order to evaluate an image quality after denoising, we used well-known criteria MSE (Mean Square Error), PSNR (Peak Signal-toNoise Ratio) and SSIM (Structure SIMilarity) (Wang, 2004; Wang, 2006). We give priority to PSNR, because it is most popular and used to evaluate the quality of restored signal in signal processing in general, and in image processing, especially.

\section{DENOISING MODEL FOR MIXED POISSON- GAUSSIAN NOISE}

Let in $\mathrm{R}^{2}$ space a bounded domain $\Omega \subset \mathrm{R}^{2}$ be given. Let us call functions $u(x, y) \in \mathrm{R}^{2}$ and $v(x, y) \in \mathrm{R}^{2}$, respectively, ideal (without noise) and observed images (noisy), where $(x, y) \in \Omega$.

If the function $u$ is smooth, then its total variation is defined by

$$
V_{T}[u]=\int_{\Omega}|\nabla u| d x d y,
$$

where $\nabla u=\left(u_{x}, u_{y}\right)$ is a gradient (nabla operator), $u_{x}=\partial u / \partial x$, $u_{y}=\partial u / \partial y,|\nabla u|=\sqrt{u_{x}^{2}+u_{y}^{2}}$. In this paper, we only consider function $u$ that always has limited total variation $V_{T}[u]<\infty$. 


\section{1}

\section{Denoising Model}

According to results (Chang, 2005; Burger, 2008; Rudin, 1992; Chen, 2013; Scherzer, 2009), image smoothness is characterised by the total variation. The total variation of noisy image is always greater than the total variation of smoothed image.

When Rudin solved the problem $V_{T}[u] \rightarrow$ min, he used this characteristic and assumed, that Gaussian noise variance is fixed by the additional constraint

$$
\int_{\Omega}(u-v)^{2} d x d y=\text { const } .
$$

He proposed the ROF model to remove Gaussian noise from an image

$$
u^{*}=\underset{u}{\arg \min }\left(\int_{\Omega}|\nabla u| d x d y+\frac{\lambda}{2} \int_{\Omega}(u-v)^{2} d x d y\right),
$$

where $\lambda>0$ is a Lagrange multiplier.

Le T. (2007) proposed another model to remove Poisson noise based on ROF model:

$$
u^{*}=\underset{u}{\arg \min }\left(\int_{\Omega}|\nabla u| d x d y+\beta \int_{\Omega}(u-v \ln (u)) d x d y\right),
$$

where $\beta$ is a regularization coefficient. We call it a modified ROF model for Poisson noise.

In order to develop the denoising model for mixed noise, we also solve the problem based on the smooth characteristic of the total variation

$$
V_{T}[u] \rightarrow \min .
$$

And we also define a constrained condition. We assume that with given image $u$, the mixed noise in image is fixed too (Poisson noise is unchangeable, and Gaussian noise only depends on noise variance):

$$
\int_{\Omega} \ln (p(v \mid u)) d x d y=\text { const },
$$

where $p(v \mid u)$ is a conditional probability.

Let us consider Gaussian noise. Its probability density function (p.d.f.) is

$$
p_{1}(v \mid u)=\exp \left(-\frac{(v-u)^{2}}{2 \sigma^{2}}\right) /(\sigma \sqrt{2 \pi}) .
$$

For Poisson noise the p.d.f. is

$$
p_{2}(v \mid u)=\frac{\exp (-u) u^{v}}{v !} .
$$

We have to note that intensity levels of image colours are integer (for example, the intensity interval for an 8-bit grayscale image is from 0 to 255), so we regard $u$ as an integer value, but this will ultimately be unnecessary (Le 2007).

In order to treat combination of Gaussian and Poisson noises, we assume the following linear combination

$$
\ln (p(v \mid u))=\lambda_{1} \ln \left(p_{1}(v \mid u)\right)+\lambda_{2} \ln \left(p_{2}(v \mid u)\right),
$$

where $\lambda_{1}>0, \lambda_{2}>0, \lambda_{1}+\lambda_{2}=1$.

According to (1), we obtain the denoising problem with constrained condition as following:

$$
\left\{\begin{array}{l}
u^{*}=\underset{u}{\arg \min } \int_{\Omega}|\nabla u| d x d y \\
\int_{\Omega}\left(\frac{\lambda_{1}}{2 \sigma^{2}}(v-u)^{2}+\lambda_{2}(u-v \ln (u))\right) d x d y=\kappa,
\end{array}\right.
$$

where $\kappa$ is a constant value.

We can transform this constrained optimization problem to the unconstrained optimization problem by using Lagrange functional

$$
\begin{gathered}
L(u, \tau)=\int_{\Omega}|\nabla u| d x d y+\tau\left(\frac{\lambda_{1}}{2 \sigma^{2}} \int_{\Omega}(v-u)^{2} d x d y+\right. \\
\left.\quad \lambda_{2} \int_{\Omega}(u-v \ln (u)) d x d y-\kappa\right)
\end{gathered}
$$

to find

$$
\left(u^{*}, \tau^{*}\right)=\underset{u, \tau}{\arg \min } L(u, \tau),
$$

where $\tau>0$ is a Lagrange multiplier.

This is our proposed model to remove mixed PoissonGaussian noise from digital image. We have to notice that, if $\lambda_{1}=0$ and $\beta=\lambda_{2} \tau$, we obtain modified ROF model for removing Poisson noise. If $\lambda_{2}=0$ and $\lambda=\lambda_{1} /\left(2 \sigma^{2}\right)$, then we obtain ROF model for removing Gaussian noise. In the case of $\lambda_{1}>0, \lambda_{2}>0$ we get the model for removing mixed PoissonGaussian noise.

\subsection{Model Discretization}

In order to solve the problem (2), we can use the Lagrange multipliers method (Zeidler, 1985; Rubinov, 2003; Gill, 1974).

However, in this paper, we will solve it by using the EulerLagrange equation (Zeidler, 1985).

Let function $f(x, y)$ be defined in limited domain $\Omega \subset \mathrm{R}^{2}$ and be the second-order continuous differentiable one by $x$ and $y$ for $(x, y) \in \Omega$.

We consider the special convex functional $F\left(x, y, f, f_{x}, f_{y}\right)$, where $f_{x}=\partial f / \partial x, f_{y}=\partial f / \partial y$.

The solution of the optimization problem

$$
\int_{\Omega} F\left(x, y, f, f_{x}, f_{y}\right) d x d y \rightarrow \min
$$

satisfies the following Euler-Lagrange equation

$$
\begin{gathered}
F_{f}\left(x, y, f, f_{x}, f_{y}\right)-\frac{\partial}{\partial x} F_{f_{x}}\left(x, y, f, f_{x}, f_{y}\right)- \\
\frac{\partial}{\partial y} F_{f_{y}}\left(x, y, f, f_{x}, f_{y}\right)=0,
\end{gathered}
$$

where

$$
F_{f}=\partial F / \partial f, F_{f_{x}}=\partial F / \partial f_{x}, F_{f_{y}}=\partial F / \partial f_{y} .
$$

We use the result above to solve the problem (2). The solution of the problem (2) is given by the following EulerLagrange equation:

$$
\begin{gathered}
-\frac{\lambda_{1}}{\sigma^{2}}(v-u)+\lambda_{2}\left(1-\frac{v}{u}\right)- \\
\mu \frac{\partial}{\partial x}\left(\frac{u_{x}}{\sqrt{u_{x}^{2}+u_{y}^{2}}}\right)-\mu \frac{\partial}{\partial y}\left(\frac{u_{y}}{\sqrt{u_{x}^{2}+u_{y}^{2}}}\right)=0, .
\end{gathered}
$$

where $\mu=1 / \tau$. We can reduce (3) to

$$
\frac{\lambda_{1}}{\sigma^{2}}(v-u)-\lambda_{2}\left(1-\frac{v}{u}\right)+
$$




$$
\mu \frac{u_{x x} u_{y}^{2}-2 u_{x} u_{y} u_{x y}+u_{x}^{2} u_{y y}}{\left(u_{x}^{2}+u_{y}^{2}\right)^{3 / 2}}=0,
$$

where

$$
\begin{gathered}
u_{x x}=\frac{\partial^{2} u}{\partial x^{2}}, u_{y y}=\frac{\partial^{2} u}{\partial y^{2}}, \\
u_{x y}=\frac{\partial}{\partial x}\left(\frac{\partial u}{\partial y}\right)=\frac{\partial}{\partial y}\left(\frac{\partial u}{\partial x}\right)=u_{y x} .
\end{gathered}
$$

In order to discretize the equation (4), we add an artificial time parameter and consider the function $u=u(x, y, t)$. Then the equation (4) relates to the following diffusion equation

$$
\begin{aligned}
& u_{t}=\frac{\lambda_{1}}{\sigma^{2}}(v-u)-\lambda_{2}\left(1-\frac{v}{u}\right)+ \\
& \mu \frac{u_{x x} u_{y}^{2}-2 u_{x} u_{y} u_{x y}+u_{x}^{2} u_{y y}}{\left(u_{x}^{2}+u_{y}^{2}\right)^{3 / 2}},
\end{aligned}
$$

where $u_{t}=\partial u / \partial t$

We can write the discretized form of the equation (5) as following:

$$
\begin{gathered}
u_{i j}^{k+1}=u_{i j}^{k}+\xi\left(\frac{\lambda_{1}}{\sigma^{2}}\left(v_{i j}-u_{i j}^{k}\right)-\right. \\
\left.\lambda_{2}\left(1-\frac{v_{i j}}{u_{i j}^{k}}\right)+\mu \varphi_{i j}^{k}\right),
\end{gathered}
$$

where

$$
\begin{gathered}
\varphi_{i j}^{k}=\frac{\nabla_{x x}\left(u_{i j}^{k}\right)\left(\nabla_{y}\left(u_{i j}^{k}\right)\right)^{2}}{\left(\left(\nabla_{x}\left(u_{i j}^{k}\right)\right)^{2}+\left(\nabla_{y}\left(u_{i j}^{k}\right)\right)^{2}\right)^{3 / 2}}+ \\
\frac{-2 \nabla_{x}\left(u_{i j}^{k}\right) \nabla_{y}\left(u_{i j}^{k}\right) \nabla_{x y}\left(u_{i j}^{k}\right)+\left(\nabla_{x}\left(u_{i j}^{k}\right)\right)^{2} \nabla_{y y}\left(u_{i j}^{k}\right)}{\left(\left(\nabla_{x}\left(u_{i j}^{k}\right)\right)^{2}+\left(\nabla_{y}\left(u_{i j}^{k}\right)\right)^{2}\right)^{3 / 2}}, \\
\nabla_{x}\left(u_{i j}^{k}\right)=\frac{u_{i+1, j}^{k}-u_{i-1, j}^{k}}{2 \Delta x}, \nabla_{y}\left(u_{i j}^{k}\right)=\frac{u_{i, j+1}^{k}-u_{i, j-1}^{k}}{2 \Delta y}, \\
\nabla_{x x}\left(u_{i j}^{k}\right)=\frac{u_{i+1, j}^{k}-2 u_{i j}^{k}+u_{i-1, j}^{k}}{(\Delta x)^{2}}, \\
\nabla_{y y}\left(u_{i j}^{k}\right)=\frac{u_{i, j+1}^{k}-2 u_{i j}^{k}+u_{i, j-1}^{k}}{(\Delta y)^{2}}, \\
u_{0 j}^{k}=u_{1 j}^{k} ; u_{N_{1}+1, j}^{k}=\frac{u_{i+1, j+1}^{k}-u_{i+1, j-1}^{k}-u_{i-1, j+1}^{k}+u_{i-1, j-1}^{k}}{4 \Delta x \Delta y}, u_{i 0}^{k}=u_{i 1}^{k} ; u_{i, N_{2}+1}^{k}=u_{i, N_{2}}^{k} ; \\
i=1, \ldots, N_{1} ; j=1, \ldots, N_{2} ; \\
k=0,1, \ldots, K ; \Delta x=\Delta y=1 ; 0<\xi<1 .
\end{gathered}
$$

Here $K$ is enough great number. In this paper, we use $K=500$.

\subsection{Finding Optimal Parameters}

We can use the procedure (6) to perform image denoising. In this procedure, values of parameters $\lambda_{1}, \lambda_{2}, \mu, \sigma$ need to be given. In some cases, we have to define these parameters to perform image denoising automatically. Then parameters $\lambda_{1}, \lambda_{2}, \mu$ in process (6) need to be changed into $\lambda_{1}^{k}, \lambda_{2}^{k}, \mu^{k}$ for each step $k$. So we obtain new procedure that allows us to calculate values of these parameters automatically in iteration steps.

\subsubsection{Optimal Parameters $\lambda_{1}$ and $\lambda_{2}$}

Let $(u, \tau)$ be a solution of the problem (2). Then we get the condition

$$
\frac{\partial L(u, \tau)}{\partial u}=0 .
$$

This condition gives us the optimal parameters $\lambda_{1}, \lambda_{2}$ :

$$
\lambda_{1}=\frac{\int_{\Omega}\left(1-\frac{v}{u}\right) d x d y}{\frac{1}{\sigma^{2}} \int_{\Omega}(v-u) d x d y+\int_{\Omega}\left(1-\frac{v}{u}\right) d x d y},
$$

Its discretized form is

$$
\begin{gathered}
\lambda_{1}^{k}=\frac{\sum_{i=1}^{N_{1}} \sum_{j=1}^{N_{2}}\left(1-\frac{v_{i j}}{u_{i j}^{k}}\right)}{\sum_{i=1}^{N_{1}} \sum_{j=1}^{N_{2}}\left(\frac{v_{i j}-u_{i j}^{k}}{\sigma^{2}}+1-\frac{v_{i j}}{u_{i j}^{k}}\right)} \\
\lambda_{2}^{k}=1-\lambda_{1}^{k},
\end{gathered}
$$

where $k=0,1, \ldots, K$.

\subsubsection{Optimal Parameter $\mu$}

In order to find an optimal parameter $\mu$, we multiply (3) by $(u-v)$ and integrate by parts over $\Omega$. Finally, we obtain the formula to find the optimal parameter $\mu$ :

$$
\mu=\frac{\int_{\Omega}\left(-\frac{\lambda_{1}}{\sigma^{2}}(u-v)^{2}-\lambda_{2} \frac{(u-v)^{2}}{u}\right) d x d y}{\int_{\Omega}\left(\sqrt{u_{x}^{2}+u_{y}^{2}}-\frac{u_{x} v_{x}+u_{y} v_{y}}{\sqrt{u_{x}^{2}+u_{y}^{2}}}\right) d x d y} .
$$

Its discretized form is

$$
\mu^{k}=\frac{\sum_{i=1}^{N_{1}} \sum_{j=1}^{N_{2}}\left(-\frac{\lambda_{1}^{k}}{\sigma^{2}}\left(u_{i j}^{k}-v_{i j}\right)^{2}-\lambda_{2}^{k} \frac{\left(u_{i j}^{k}-v_{i j}\right)^{2}}{u_{i j}^{k}}\right)}{\sum_{i=1}^{N_{1}} \sum_{j=1}^{N_{2}} \eta_{i j}^{k}},
$$

where

$$
\begin{gathered}
\eta_{i j}^{k}=\sqrt{\left(\nabla_{x}\left(u_{i j}^{k}\right)\right)^{2}+\left(\nabla_{y}\left(u_{i j}^{k}\right)\right)^{2}}- \\
\frac{\nabla_{x}\left(u_{i j}^{k}\right) \nabla_{x}\left(v_{i j}\right)+\nabla_{y}\left(u_{i j}^{k}\right) \nabla_{y}\left(v_{i j}\right)}{\sqrt{\left(\nabla_{x}\left(u_{i j}^{k}\right)\right)^{2}+\left(\nabla_{y}\left(u_{i j}^{k}\right)\right)^{2}}}, \\
\nabla_{x}\left(u_{i j}^{k}\right)=\frac{u_{i+1, j}^{k}-u_{i-1, j}^{k}}{2 \Delta x}, \nabla_{y}\left(u_{i j}^{k}\right)=\frac{u_{i, j+1}^{k}-u_{i, j-1}^{k}}{2 \Delta y}, \\
\nabla_{x}\left(v_{i j}^{k}\right)=\frac{v_{i+1, j}^{k}-v_{i-1, j}^{k}}{2 \Delta x} v, \nabla_{y}\left(v_{i j}^{k}\right)=\frac{v_{i, j+1}^{k}-v_{i, j-1}^{k}}{2 \Delta y}, \\
u_{0 j}^{k}=u_{1 j}^{k} ; u_{N_{1}+1, j}^{k}=u_{N_{1}, j}^{k} ; u_{i 0}^{k}=u_{i 1}^{k} ; u_{i, N_{2}+1}^{k}=u_{i, N_{2}}^{k} ; \\
v_{0 j}=v_{1 j} ; v_{N_{1}+1, j}=v_{N_{1} j} ; v_{i 0}=v_{i 1} ; v_{i, N_{2}+1}=v_{i, N_{2}} ;
\end{gathered}
$$




$$
i=1, \ldots, N_{1} ; j=1, \ldots, N_{2} ; k=0,1, \ldots, K ; \Delta x=\Delta y=1 .
$$

\subsubsection{Optimal Parameter $\sigma$}

In order to evaluate this parameter $\sigma$, we use the result of Immerker (1996):

$$
\begin{gathered}
\sigma=\frac{\sqrt{\pi / 2}}{6\left(N_{1}-2\right)\left(N_{2}-2\right)} \sum_{i=1}^{N_{1}} \sum_{j=1}^{N_{2}}\left|u_{i j} * \Lambda\right|, \text { where } \\
\Lambda=\left(\begin{array}{ccc}
1 & -2 & 1 \\
-2 & 4 & -2 \\
1 & -2 & 1
\end{array}\right) \text { is the mask of an image. }
\end{gathered}
$$

Operator $*$ is a convolution operator, where

$$
\begin{gathered}
u_{i j} * \Lambda=u_{i-1, j-1} \Lambda_{33}+u_{i, j-1} \Lambda_{32}+u_{i+1, j-1} \Lambda_{31}+u_{i-1, j} \Lambda_{23}+ \\
u_{i j} \Lambda_{22}+u_{i+1, j} \Lambda_{21}+u_{i-1, j+1} \Lambda_{13}+u_{i, j+1} \Lambda_{12}+u_{i+1, j+1} \Lambda_{11}, \\
i=1, \ldots, N_{1} ; j=1, \ldots, N_{2} ; \\
u_{i j}=0, \text { if } i=0, \text { or } j=0, \text { or } i=N_{1}+1, \\
\text { or } j=N_{2}+1 .
\end{gathered}
$$

We have to notice, that the parameter $\sigma$ is just evaluated at first time of the iteration process.

\subsection{Image Quality Evaluation}

In order to evaluate image quality after denoising, we use criteria MSE (Mean Square Error), PSNR (Peak Signal-toNoise Ratio) and SSIM (Structure SIMilarity) (Wang 2004, 2006):

$$
\begin{gathered}
Q_{M S E}=\frac{1}{N_{1} N_{2}} \sum_{i=1}^{N_{1}} \sum_{j=1}^{N_{2}}\left(u_{i j}-v_{i j}\right)^{2}, \\
Q_{P S N R}=10 \lg \left(\frac{N_{1} N_{2} L^{2}}{\sum_{i=1}^{N_{1}} \sum_{j=1}^{N_{2}}\left(u_{i j}-v_{i j}\right)^{2}}\right), \\
Q_{S S I M}=\frac{\left(2 \bar{u} \bar{v}+C_{1}\right)\left(2 \sigma_{u v}+C_{2}\right)}{\left(\bar{u}^{2}+\bar{v}^{2}+C_{1}\right)\left(\sigma_{u}^{2}+\sigma_{v}^{2}+C_{2}\right)},
\end{gathered}
$$

where

$$
\begin{gathered}
\bar{u}=\frac{1}{N_{1} N_{2}} \sum_{i=1}^{N_{1}} \sum_{j=1}^{N_{2}} u_{i j}, \bar{v}=\frac{1}{N_{1} N_{2}} \sum_{i=1}^{N_{1}} \sum_{j=1}^{N_{2}} v_{i j} . \\
\sigma_{u}^{2}=\frac{1}{N_{1} N_{2}-1} \sum_{i=1}^{N_{1}} \sum_{j=1}^{N_{2}}\left(u_{i j}-\bar{u}\right)^{2}, \\
\sigma_{v}^{2}=\frac{1}{N_{1} N_{2}-1} \sum_{i=1}^{N_{1}} \sum_{j=1}^{N_{2}}\left(v_{i j}-\bar{v}\right)^{2}, \\
\sigma_{u v}=\frac{1}{N_{1} N_{2}-1} \sum_{i=1}^{N_{1}} \sum_{j=1}^{N_{2}}\left(u_{i j}-\bar{u}\right)\left(v_{i j}-\bar{v}\right), \\
C_{1}=\left(K_{1} L\right)^{2}, C_{2}=\left(K_{2} L\right)^{2} ; K_{1}<<1 ; K_{2}<<1 .
\end{gathered}
$$

For example, $K_{1}=K_{2}=10^{-6}, L$ is image intensity, where, for example, $L=2^{8}-1=255$ for an 8 -bit greyscale images.

The greater value $Q_{P S N R}$, the better image quality. If $Q_{P S N R}$ is between 20 and 25 , then an image quality is acceptable, for example, for the wireless transmission (Thomos, 2006).

$Q_{S S I M}$ is used to evaluate image quality by comparing similarity of both images. Its value is between -1 and 1 . The greater value $Q_{S S I M}$, the better image quality.

$Q_{M S E}$ is a criterion to evaluate the difference between two images. $Q_{M S E}$ is mean-squared error. The lower value $Q_{M S E}$, the better restoration result. The value of $Q_{M S E}$ also relates to the value of $Q_{P S N R}$.

\section{$2.5 \quad$ Initial solution}

Because the iteration process uses the initial solution to perform finding solution, so the restoration result of automatically evaluated parameters case also depends on this initial solution. This dependency affects to restoration result, but it is not too much.

The initial solution can be given by one from two methods: directly given or given through a set of initial parameters $\left(\lambda_{1}^{0}, \lambda_{2}^{0}, \mu^{0}\right)$.

If the initial values of parameters $\lambda_{1}, \lambda_{2}, \mu$ are given, the obtained solution is not very good. Because when we set up the initial parameters to find initial solution, the priority of processing of Gaussian and Poisson noise on initial solution is fixed and the result will depend on these initial parameters.

If the initial solution is constant value, the total variation and the differences by $x$-direction and $y$-direction will be 0 . This is very bad for our iteration process.

If we make an artificial image by randomizing, the restoration result is bad. Because the random function will affect to the noise property.

The best case is the initial solution need to be enough different with noisy image but not much. In experiment, we make this solution by using average neighbour pixels (closed similar with noise assessment method of Immerker).

\subsection{Experiments}

We use an example to test our model in the case of processing a real image. In this case, we use an image of human skull with the size 300x300 pixel (Figure 1a). We zoom, crop and show the part of the original image under processing (Figure $1 b-1 \mathrm{f}$ ).

First, we create the noisy image by adding Gaussian noise (Figure 1c) and second, create noisy image by adding Poisson noise (Figure 1d).

In order to calculate proportion between intensities of Gaussian and Poisson noises, we calculate the variance of Poisson noise. The value of variance of Gaussian noise is calculated via Poisson noise variance. Let the variance of Gaussian noise be four times greater than the variance of Poisson noise.

First, let us consider Poisson noise. Its distribution is $p_{2}(v \mid u)$, value of the variance of Poisson noise is $\sigma_{2}=\sqrt{u_{i j}}$, respectively, with $u_{i j}$ at every pixel $(i, j)$ of image, where $i=1, \ldots, N_{1} ; j=1, \ldots, N_{2}$. We denote this Poisson noisy image as $v^{(2)}$. Obviously, intensity value of $v^{(2)}$ ought to be between 0 and 255. If the intensity value of some pixels are out of this interval, they need to be reset to intensity value of respective pixel of the original image $u$, that means $v^{(2)}{ }_{i j}=u_{i j}$. 
In this case, number of them is $5(0.0056 \%)$. The variance of Poisson noise can be calculated as average value $\sigma_{2}=10.0603$.

Now, we consider Gaussian noise. Its variance need to be 40.2412 (because we explained above, variance of Gaussian noise is four times over variance of Poisson noise). We denote this Gaussian noisy image as $v^{(1)}$. As above case, intensity value of $v^{(1)}$ also need to be between 0 and 255. In this case, there are 5780 pixels out of this interval, respectively $6.42 \%$ of all image pixels.

We create resulting noisy image (Figure 1e) by combining first noisy and second noisy images with proportion 0.5 for Gaussian noisy image $v^{(1)}$ and 0.5 for Poisson noisy image $v^{(2)}$.

This means $v=0.5 v^{(1)}+0.5 v^{(2)}$. Hence:

$$
\lambda_{1} / \lambda_{2}=\frac{40.2412 \cdot 0.5}{10.0603 \cdot 0.5}=4 / 1 \text {. }
$$

As a result: $\lambda_{1}=4 / 5=0.8, \lambda_{2}=1 / 5=0.2$.

Values of $Q_{M S E}, Q_{P S N R}$ and $Q_{S S I M}$ of noisy image are respectively $427.9526,21.4168$, and 0.4246 .

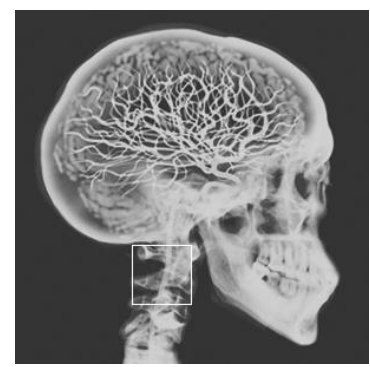

a)

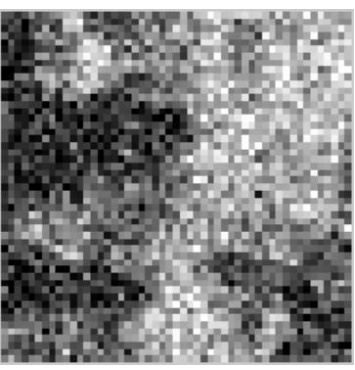

c)

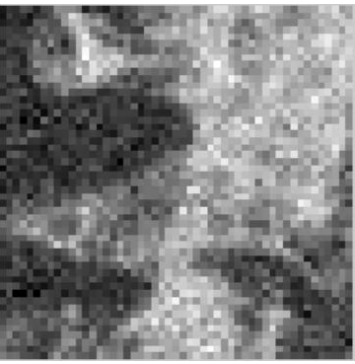

e)

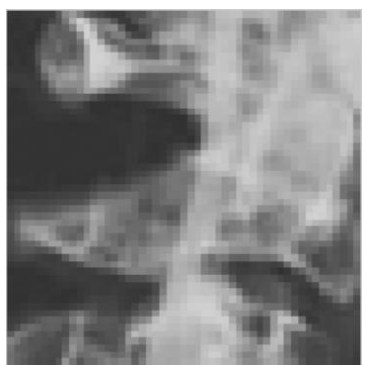

b)

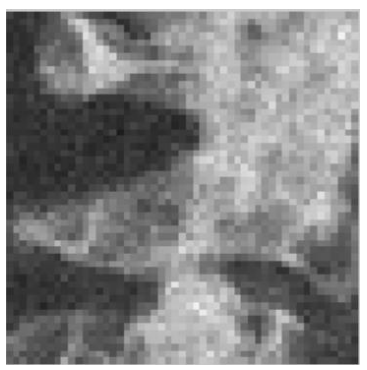

d)

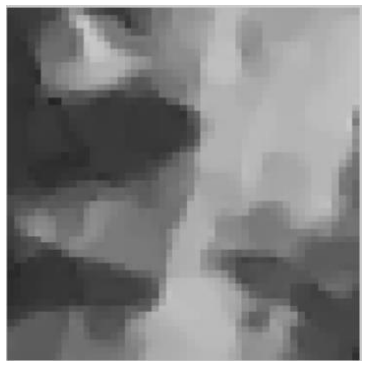

f)
Figure 1. Denoising of real image: a) original image, b) cropped image, c) with Gaussian noise, d) with Poisson noise, e) with mixed noise, f) after denoising.

\begin{tabular}{|c|c|c|c|}
\hline & $Q_{P S N R}$ & $Q_{S S I M}$ & $Q_{M S E}$ \\
\hline Noisy & 21.4168 & 0.4246 & 427.9526 \\
\hline ROF & 26.5106 & 0.8465 & 145.2183 \\
\hline Median & 25.6477 & 0.7871 & 177.1364 \\
\hline Wiener & 24.2657 & 0.6596 & 243.5077 \\
\hline $\begin{array}{c}\text { Proposed } \\
\text { method with } \\
\lambda_{1}=0.8, \\
\lambda_{2}=0.2, \\
\mu=0.0857, \\
\sigma=40.2412 .\end{array}$ & & & \\
\hline $\begin{array}{c}\text { Proposed } \\
\text { method with } \\
\text { evaluated } \\
\text { parameters } \\
\lambda_{1}=0.8095, \\
\lambda_{2}=0.1905, \\
\mu=0.0970, \\
\sigma=38.2310 .\end{array}$ & 27.4315 & 0.8198 & $\mathbf{1 1 7 . 4 7 1 3}$ \\
& & & \\
\hline
\end{tabular}

Table 1. Quality comparison of noise removal methods for real image of human skull.

The Table 1 shows the result of denoising for real image in cases: given parameters and automatically evaluated parameters.

We have to notice, that in the case of the real image, the value of $Q_{P S N R}$ of denoising for given ideal parameters is better, than the value of $Q_{P S N R}$ of denoising for automatically evaluated parameters, but the value of $Q_{S S I M}$ is inversed.

Based on experimental results, we can see that the restoration result of automatically evaluated parameters case depends on initial solution.

We use convolution operator to make a new image. The Table 2 shows the dependency of restoration result on initial solution, where:

(a) Initial parameters $\lambda_{1}^{0}=0, \lambda_{2}^{0}=1, \mu=1$;

(b) Initial parameters $\lambda_{1}^{0}=\lambda_{2}^{0}=0.5, \mu=1$;

(c) Initial solution $u^{0}=\operatorname{rand}(\cdot, \cdot)$ with $\operatorname{rand}(\cdot, \cdot)$ to create randomized two-dimensional matrix with specific size;

(d) Initial solution $u^{0}=v * \mathrm{~A}$ with $A=\frac{1}{9}\left(\begin{array}{lll}1 & 1 & 1 \\ 1 & 1 & 1 \\ 1 & 1 & 1\end{array}\right)$.

Table 2 shows the best denoising result for the case (d) with respect two most important criteria (PSNR and MSE) .

\begin{tabular}{|c|c|c|c|c|}
\hline & (a) & (b) & (c) & (d) \\
\hline$\lambda_{1}$ & 0.8095 & 0.8114 & 0.9256 & 0.8069 \\
\hline$\lambda_{2}$ & 0.1905 & 0.1886 & 0.0744 & 0.1931 \\
\hline$\mu$ & 0.0970 & 0.0985 & 0.1026 & 0.0965 \\
\hline$\sigma$ & \multicolumn{4}{|c|}{38.2310} \\
\hline$Q_{P S N R}$ & 27.2567 & 27.1327 & 26.4279 & $\mathbf{2 7 . 2 5 7 1}$ \\
\hline$Q_{M S E}$ & 122.2941 & 125.8371 & 148.0081 & $\mathbf{1 2 1 . 6 3 2 0}$ \\
\hline$Q_{S S I M}$ & 0.8383 & 0.8381 & $\mathbf{0 . 8 4 9 7}$ & 0.8384 \\
\hline
\end{tabular}

Table 2. Dependency of restoration result on initial solution. 


\section{CONCLUSIONS}

In this paper, we proposed the method that is based on variational approach to remove combination of Poisson and Gaussian noises (mixed noise).

The denoising result depends on parameters, especially on coefficients of linear combination $\lambda_{1}$ and $\lambda_{2}$. We can specify the values of parameters or these values can be automatically evaluated. In order to apply this model to real image, we need to use the proposed method with automatically evaluated parameters. In this case, the solution also depends on initial solution.

The proposed method can be applied to remove separate Gaussian or Poisson noise (respectively ROF model and modified ROF model for Poisson noise), or mixed PoissonGaussian noise as well.

We also can use this variational approach to remove other kinds of noise, such as noise of magnetic resonance images (MRI), ultrasonogram, etc.

\section{REFERENCES}

Abe C., Shimamura T., 2012, 'Iterative Edge-Preserving adaptive Wiener filter for image denoising', ICCEE, vol. 4, no. 4, pp. 503-506.

Burger M., 2008, Level set and PDE based reconstruction methods in imaging, Springer.

Caselles V., Chambolle A., Novaga M., 2011, Handbook of mathematical methods in imaging, Springer.

Chan T.F., Shen J., 2005, Image processing and analysis: Variational, PDE, Wavelet, and stochastic methods, SIAM.

Chambolle A., 2009, 'An introduction to total variation for image analysis', Theoretical foundations and numerical methods for sparse recovery, vol. 9, pp. 263-340.

Chen K., 2013, 'Introduction to variational image processing models and application', International journal of computer mathematics, vol. 90, no. 1, pp. 1-8.

Getreuer P., 2012, 'Rudin-Osher-Fatemi total variation denoising using split Bregman'. IPOL 2012, 'http://www.ipol.im/pub/art/2012/g-tvd/'.

Gill P.E., Murray W., 1974, Numerical methods for constrained optimization, Academic Press Inc.

Immerker J., 1996, 'Fast noise variance estimation', Computer vision and image understanding, vol. 64, no.2, pp. 300-302.

Jezierska A., 2012, 'Poisson-Gaussian noise parameter estimation in fluorescence microscopy imaging', IEEE International Symposium on Biomedical Imaging $9^{\text {th }}$, pp. 16631666.

Jezierska A., 2011, 'An EM approach for Poisson-Gaussian noise modelling', EUSIPCO 19 $9^{\text {th }}$, vol. 62, is. 1, pp. 13-30.

Le T., Chartrand R., Asaki T.J., 2007, 'A variational approach to reconstructing images corrupted by Poisson noise', Journal of mathematical imaging and vision, vol. 27, is. 3, pp. 257-263.
Li F., Shen C., Pi L., 2006, 'A new diffusion-based variational model for image denoising and segmentation', Journal mathematical imaging and vision, vol. 26, is. 1-2, pp. 115-125.

Luisier F., Blu T., Unser M., 2011, 'Image denoising in mixed Poisson-Gaussian noise', IEEE transaction on Image processing, vol. 20, no. 3, pp. 696-708.

Lysaker M., Tai X., 2006, 'Iterative image restoration combining total variation minimization and a second-order functional', International journal of computer vision, vol. 66, pp. 5-18.

Nick V., 2009, Getty images, 'http://well.blogs.nytimes.com/2009/09/16/what-sort-ofexercise-can-make-you-smarter/'.

Rankovic N., Tuba M., 2012, 'Improved adaptive median filter for denoising ultrasound images', Advances in computer science, WSEAS ECC'12, pp. 169-174.

Rubinov A., Yang X., 2003, Applied Optimization: Lagrangetype functions in constrained non-convex optimization, Springer.

Rudin L.I., Osher S., Fatemi E., 1992, 'Nonlinear total variation based noise removal algorithms', Physica D. vol. 60, pp. 259268.

Scherzer O., 2009, Variational methods in Imaging, Springer.

Thomos N., Boulgouris N.V., Strintzis M.G., 2006, 'Optimized Transmission of JPEG2000 streams over Wireless channels', IEEE transactions on image processing, vol. 15, no.1, pp .5467.

Tran M.P., Peteri R., Bergounioux M., 2012, 'Denoising 3D medical images using a second order variational model and wavelet shrinkage', Image analysis and recognition, vol. 7325, pp. 138-145.

Wang C., Li T., 2012, 'An improved adaptive median filter for Image denoising’, ICCEE, vol. 53, no. 2.64, pp. 393-398.

Wang Z., 2004, 'Image quality assessment: From error visibility to structural similarity', IEEE transaction on Image processing, vol. 13 , no. 4 , pp. 600-612.

Wang Z., Bovik A.C., 2006, Modern image quality assessment, Morgan \& Claypool Publisher.

Xu J., Feng X., Hao Y., 2014, 'A coupled variational model for image denoising using a duality strategy and split Bregman', Multidimensional systems and signal processing, vol. 25, pp. 83-94.

Revised January 2015 